\title{
A Low Dissipation Method to Cure the Grid-Aligned Shock Instability
}

\author{
Nico Fleischmann ${ }^{\mathrm{a}, *}$, Stefan Adami ${ }^{\mathrm{a}}$, Xiangyu Y. Hu ${ }^{\mathrm{a}}$, Nikolaus A. Adams ${ }^{\mathrm{a}}$ \\ ${ }^{a}$ Technical University of Munich, Department of Mechanical Engineering, Chair of \\ Aerodynamics and Fluid Mechanics, Boltzmannstraße 15, 85748 Garching, Germany
}

\section{Abstract}

The grid-aligned shock instability prevents an accurate computation of high Mach number flows using low-dissipation shock-capturing methods. In particular one manifestation, the so-called carbuncle phenomenon, has been investigated by various different groups over the past decades. Nevertheless, the mechanism of this instability is still not fully understood and commonly is suppressed by the introduction of additional numerical dissipation. However, present approaches may either significantly deteriorate the resolution of complex flow evolutions or involve additional procedures to limit stabilization measures to the shock region.

Instead of increasing the numerical dissipation, in this paper, we present an alternative approach that relates the problem to the low Mach number in transverse direction of the shock front. We show that the inadequate scaling of the acoustic dissipation in the low Mach number limit is the prime reason for the instability. Our approach is to increase the "numerical" Mach

\footnotetext{
${ }^{*}$ Corresponding author.

Email addresses: nico.fleischmann@tum.de (Nico Fleischmann), stefan.adami@aer.mw.tum.de (Stefan Adami), xiangyu.hu@tum.de (Xiangyu Y. Hu), nikolaus. adams@tum.de (Nikolaus A. Adams)
} 
number locally whenever the advection dissipation is small compared to the acoustic dissipation. A very simple modification of the eigenvalue calculation in the Roe approximation leads to a scheme with less numerical dissipation than the original Roe flux which prevents the grid-aligned shock instability. The simplicity of the modification allows for a detailed investigation of multidimensional effects. By showing that modifications in flow direction affect the shock stability in the transverse directions we confirm the multidimensional nature of the instability. The efficiency and robustness of the modified scheme is demonstrated for a wide range of test cases that are known to be particularly prone to the shock instability. Moreover, the modified flux also is successfully applied to multi-phase flows.

Keywords: shock instability, carbuncle phenomenon, low Mach number effect, low-dissipation schemes, WENO, shock-capturing

\section{Introduction}

Modern applications of computational fluid dynamics involve complex interactions across scales such as shock interactions with turbulent structures and multiphase interfaces $[1,2]$. In the last decades, a variety of different numerical approaches were developed aiming at schemes that combine high accuracy and high robustness [3]. Shock capturing methods based on Godunov's approach [4] nowadays are among the most successful methods for simulating compressible flows involving shock waves and other discontinuities. Their versatility and ease of implementation make them advantageous over shock tracking methods, especially when new shock waves emerge during the simulation. Following Godunov's approach [4], cell-face fluxes are deter- 
mined by Riemann problems, which can be solved locally. The popularity of the method is based on its straightforward extension to high-order reconstruction schemes, such as ENO [5] or WENO [6] schemes. Originally the exact Riemann solution was computed iteratively, which soon was replaced by more efficient approximate Riemann solvers.

Numerous approximate flux formulations have been developed in the past. They can be categorized roughly as complete and incomplete. Incomplete fluxes, such as e.g. HLL [7] or HLLE [8] solver, typically are more robust, at the expense of allowing only for a limited number of waves. Contact discontinuities usually are not captured explicitly, which often leads to unacceptable smearing of contact lines. Complete fluxes model explicitly each of the relevant waves of the underlying problem, e.g. for the three-dimensional Euler equations five waves are modelled including the contact discontinuity. Popular examples of complete Riemann approximations are HLLC [9], the Osher approximation [10] and the Roe approximation [11]. Upon combining complete Riemann solvers with high-order reconstruction schemes a minimum amount of numerical dissipation can be obtained. Such a low numerical dissipation is crucial for the accurate calculation of flow instabilities and wave transport. Despite the enormous effort spent on the development of approximate Riemann solvers, which enables the accurate simulation of various complex flow configurations, the design of an all-purpose low-dissipation high-resolution Riemann solver remains to be a challenge.

It turned out that a promising group of complete Riemann solvers fails spectacularly on certain flow configurations involving supersonic shock waves [12]. Since Peery and Imlay [13] first described their observation on the fail- 
ure of the Roe flux approximation when applied to supersonic flows around a blunt body, intense research was devoted to discovering reasons and cures for this undesirable behavior. An instability, first reported in [13], leads to a characteristic deformation of the shock front which was called "carbuncle phenomenon" due to its excrescence-like appearance. The carbuncle phenomenon ruined many efforts to compute grid-aligned shock waves using low-dissipation upwind schemes. Quirk [12] presented a catalogue of cases where Godunov type schemes were known to fail. Three of the problems he reported, namely the carbuncle phenomenon, the kinked Mach stem of a double Mach reflection and a newly described odd-even decoupling problem have in common that they develop most prominently when a high Mach number shock wave propagates aligned with the computational grid. Quirk [12] noticed that some schemes possessing good shock capturing properties are more likely to be affected by such instabilities than more dissipative flux formulations, such as the HLLE Riemann solver. Quirk [12] concluded that insufficient dissipation at the contact line might be the reason for the instability. A large number of scientific publications since have addressed various aspects of the challenging problem. Although not being fully understood, it is generally believed that the prime reason for the shock instability is insufficient numerical dissipation in the region of the shock front.

The most popular approach to cure the instability is to increase locally the numerical dissipation of the underlying scheme. Peery and Imlay [13] achieved such a stabilization by smoothing the Roe eigenvalues, resulting in a bow shock smearing over several cells. Quirk [12] noted that the ad-hoc application of Harten's entropy fix to increase numerical dissipation of the 
contact and shear waves cures the problem, has, however, no physical justification. Sanders et al. [14] performed a linear analysis that revealed that the instability is caused by an insufficient cross-flow dissipation. They proposed a multi-dimensional modification to the Roe solver, where they apply an entropy fix that depends on neighboring interfaces. Due to the spatial arrangement of the incorporated interfaces the method is called H-correction. Later, Pandolfi and D'Ambrosio [15] modified the H-correction for the application to high-speed flow boundary layers. Both the analyses of Gressier and Moschetta [16] and Pandolfi and D'Ambrosio [15] investigated the behavior of different families of approximate Riemann solvers concerning the shock instability. They concluded that schemes that explicitly capture a contact discontinuity are always to some extend prone to the grid-aligned shock instability. Liou [17] found that the dissipative pressure term in the mass flux is responsible for the instability, which motivated him to design new schemes that are both stable and able to capture contact discontinuities. The conclusion of [17] that schemes with a mass flux that is independent of the pressure term are not affected by the carbuncle phenomenon (Liou's conjecture) was questioned by other authors $[16,15,18]$. They provided counterexamples including the $\mathrm{AUSM}^{+}$scheme [19]. Ren [20] developed a shock-stable scheme based on a rotated Roe flux formulation, which automatically introduces artificial dissipation in the relevant regions. Although his approach does not require an explicit detection procedure, there is a computational overhead since the Riemann problem has to be solved twice on every cell interface. Kim et al. [21] introduced an improved Roe scheme that is capable of accurately resolving contact discontinuities and is free of the shock instability. 
The authors of [21] introduced a Mach-number-based function that controls the feeding rate of pressure fluctuation into the numerical mass flux. More recently, Chen et al. [22] followed a similar approach to stabilize the Roe scheme by introducing shear viscosity into the momentum flux, controlled by a pressure-based sensing function to constrain the effect to the shock region. They noticed that the instability of the Roe scheme may not be caused by the pressure difference, but by inadequate shear viscosity. All procedures described so far have in common that they increase the dissipation of the Roe scheme in one way or another. Similar procedures have been developed for other types of Riemann solvers, e.g. for HLLC [23, 24, 25] or AUSM [26, 27].

An alternative approach are hybrid schemes where Riemann solvers of different families are combined. The appropriate scheme is chosen locally based on a control function that evaluates the local flow condition. Quirk [12] combined a Roe scheme with an HLL scheme and obtained good results. However, the control function involves problem-dependent parameter calibration and the overall scheme still is stabilized by local application of a more dissipative scheme. Rodionov [28] argued that the exact Riemann solver is physically the most consistent way to determine inviscid fluxes, however, the introduction of additional numerical dissipation moves the approximate solution further apart from the exact Riemann problem. Thus, he presented a new approach where the problem is not solved by corrections to the Riemann solver but by introducing an artificial viscosity similar to the molecular viscosity present in the Navier-Stokes equations. This approach is independent of the applied Riemann solver.

The present work relates the grid-aligned shock instability to another well- 
known difficulty of Godunov-type schemes in the low Mach number limit. Guillard et al. [29, 30] performed an asymptotic analysis in terms of powers of the Mach number, which revealed an incorrect scaling behavior of pressure fluctuations when Godunov schemes are applied at vanishing Mach number. For a shock wave in the solution of a multi-dimensional high-Mach-number flow propagating in one direction of the underlying Cartesian grid, the disturbances parallel to the shock front travel at a low relative Mach number. For the Roe flux formulation the dissipative flux term in this case is dominated by the acoustic contribution, while the dissipation due to advection vanishes. We show two possible solution strategies to balance both contributions, and thus increase the Mach number for the calculation of the eigenvalues that determine the dissipative flux term. A locally decreased acoustic dissipation leads to an overall decrease of dissipation of the numerical scheme, while a locally increased advection dissipation leads to an overall increase of dissipation. The fundamental difference between our approach as opposed to previous attempts is that we cure the grid-aligned shock instability by a local decrease of dissipation. The implementation of our method into an existing Riemann solver is straightforward as it amounts to only few additional lines of code.

The remainder of the paper is organized as follows. In Section 2, the governing equations and the basic principles of Godunov-type methods are reviewed together with the flux formulations of the Roe approximation and a componentwise local Lax-Friedrichs approximation. The modifications made on the original schemes considering the low Mach number effect are presented in detail in Section 3. In Section 4, Quirk's odd-even decoupling case is inves- 
tigated both qualitatively and quantitatively to demonstrate the robustness of the modified Roe flux. A demonstration of the multidimensional character of the carbuncle phenomenon is presented in Section 5. Finally in Section 6, the effectiveness of the low Mach number treatment is demonstrated for the full set of relevant test cases that are commonly referred to by literature addressing the grid-aligned shock instability. Conclusions are drawn in Section 7.

\section{Governing Equations and Numerical Approach}

We consider an inviscid compressible flow that evolves according to the three-dimensional Euler equations

$$
\mathbf{U}_{t}+\mathbf{F}(\mathbf{U})_{x}+\mathbf{G}(\mathbf{U})_{y}+\mathbf{H}(\mathbf{U})_{z}=0
$$

where $\mathbf{U}$ is the density of the conserved quantities mass $\rho$, momentum $\rho \mathbf{v} \equiv$ $(\rho u, \rho v, \rho w)$ and total energy $E=\rho e+\frac{1}{2} \rho \mathbf{v}^{2}$, with $e$ being the internal energy

per unit mass. The fluxes $\mathbf{F}, \mathbf{G}$ and $\mathbf{H}$ are defined as

$$
\mathbf{F}=\left(\begin{array}{c}
\rho u \\
\rho u^{2}+p \\
\rho u v \\
\rho u w \\
u(E+p)
\end{array}\right), \mathbf{G}=\left(\begin{array}{c}
\rho v \\
\rho u v \\
\rho v^{2}+p \\
\rho v w \\
v(E+p)
\end{array}\right), \mathbf{H}=\left(\begin{array}{c}
\rho w \\
\rho u w \\
\rho v w \\
\rho w^{2}+p \\
w(E+p)
\end{array}\right)
$$

The set of equations is closed by the ideal-gas equation of state, where the pressure $p$ is given by $p=(\gamma-1) \rho e$ with a constant ratio of specific heats $\gamma$. 


\subsection{Finite volume approach}

We apply Godunov's approach approach for finite volumes to solve this set of equations numerically. This procedure is widely used for hyperbolic equations. The time evolution of the vector of cell-averaged conservative states $\overline{\mathbf{U}}$ is given by

$$
\begin{aligned}
& \frac{d}{d t} \overline{\mathbf{U}}_{i}=\frac{1}{\Delta x}\left(\mathbf{F}_{i-\frac{1}{2}, j, k}-\mathbf{F}_{i+\frac{1}{2}, j, k}+\right. \\
&\left.\mathbf{G}_{i, j-\frac{1}{2}, k}-\mathbf{G}_{i, j+\frac{1}{2}, k}+\mathbf{H}_{i, j, k-\frac{1}{2}}-\mathbf{H}_{i, j, k+\frac{1}{2}}\right),
\end{aligned}
$$

where $\mathbf{F}, \mathbf{G}$ and $\mathbf{H}$ approximate the cell-face fluxes in $x$-, $y$ - and $z$-direction, respectively. These fluxes are determined dimension-by-dimension from an approximate Riemann solver combined with a fifth-order WENO [6] spatial reconstruction scheme. Additional volume source terms, such as gravitational acceleration, are omitted here for simplicity. The resulting ODE (3) is integrated in time using a third-order strong stability-preserving (SSP) Runge-Kutta scheme [31].

\subsection{Approximate Riemann solvers}

The majority of state-of-the-art methods relies on approximate Riemann solvers since exact Riemann solvers are computationally expensive. Moreover, exact Riemann solvers are not helpful to suppress the carbuncle phenomenon since they are likewise affected by the shock instability [12]. Here, we focus on two approximate flux formulations, the classical Roe flux [11] and a componentwise local Lax-Friedrichs flux [6]. The Roe flux formulation is known to be particularly vulnerable to the carbuncle phenomenon. Its low numerical dissipation and its ability to capture contact waves accurately is generally believed to be the reason for this deficiency. The componentwise 
local Lax-Friedrichs flux is only slightly more dissipative and therefore similarly affected by the instability as the numerical examples in Sec. 4 and Sec. 6 demonstrate. Although both the Roe flux and the componentwise local Lax-Friedrichs flux are not positivity preserving in general, the latter one delivers numerically stable results in many flow conditions, where the Roe flux fails. In the following, only the $x$-direction is discussed. Due to the dimension-by-dimension approach, the other directions are handled the same way.

\subsubsection{Roe Riemann flux}

The classical Roe formulation gives the following numerical flux function

$$
\mathbf{F}_{i+1 / 2}^{R o e}=\frac{1}{2}\left(\mathbf{F}_{i+1}+\mathbf{F}_{i}\right)-\frac{1}{2} \mathbf{R}_{i+1 / 2}\left|\Lambda_{i+1 / 2}\right| \mathbf{R}_{i+1 / 2}^{-1}\left(\mathbf{U}_{i+1}-\mathbf{U}_{i}\right)
$$

where $\mathbf{R}$ and $\mathbf{R}^{-1}$ are the right and the left eigenvector matrices of the Jacobian $\partial \mathbf{F} / \partial \mathbf{U}$, and $\boldsymbol{\Lambda}$ is the diagonal matrix formed with the eigenvalues

$$
\lambda_{1}=\hat{u}-\hat{c}, \quad \lambda_{2,3,4}=\hat{u}, \quad \lambda_{5}=\hat{u}+\hat{c} .
$$

Quantities with hat-notation “?” denote density-based Roe averages

$$
\hat{k}=\frac{\bar{k}_{i} \cdot \sqrt{\rho_{i}}+\bar{k}_{i+1} \cdot \sqrt{\rho_{i+1}}}{\sqrt{\rho_{i}}+\sqrt{\rho_{i+1}}}
$$

where $k$ has to be replaced by the quantity of interest, e.g. $u$. $\bar{k}_{i}$ and $\bar{k}_{i+1}$ are the cell-averaged variables of the cells adjacent to the cell face $i+1 / 2$. No entropy-fix is applied for all computations in this paper. 
The high-order spatial WENO reconstruction is performed in characteristic space using a finite difference formulation both on

$$
\mathbf{F}_{i+1 / 2}^{+}=\mathbf{F}_{i}+\mathbf{R}_{i+1 / 2}\left|\boldsymbol{\Lambda}_{i+1 / 2}\right| \mathbf{R}_{i+1 / 2}^{-1} \mathbf{U}_{i}
$$

and

$$
\mathbf{F}_{i+1 / 2}^{-}=\mathbf{F}_{i+1}-\mathbf{R}_{i+1 / 2}\left|\Lambda_{i+1 / 2}\right| \mathbf{R}_{i+1 / 2}^{-1} \mathbf{U}_{i+1}
$$

The final flux is obtained by

$$
\mathbf{F}_{i+1 / 2}^{R o e}=\frac{1}{2}\left(\mathbf{F}_{i+1 / 2}^{+}+\mathbf{F}_{i+1 / 2}^{-}\right)
$$

\subsubsection{Componentwise local Lax-Friedrichs flux}

The only difference between the Roe flux and the componentwise local Lax-Friedrichs flux (cLLF) is the choice of eigenvalues $\boldsymbol{\Lambda}$ in Eq. (4). While density-based Roe averages are applied for the Roe flux, the eigenvalues in the componentwise local Lax-Friedrichs flux are determined by

$$
\begin{aligned}
& \left|\lambda_{1}\right|=\max \left(\left|u_{i}-c_{i}\right|,\left|u_{i+1}-c_{i+1}\right|\right), \\
& \left|\lambda_{2,3,4}\right|=\max \left(\left|u_{i}\right|,\left|u_{i+1}\right|\right) \\
& \left|\lambda_{5}\right|=\max \left(\left|u_{i}+c_{i}\right|,\left|u_{i+1}+c_{i+1}\right|\right) .
\end{aligned}
$$

Note that $\mathbf{R}$ and $\mathbf{R}^{-1}$ are identical for both flux formulations. Compared to the original local Lax-Friedrichs flux, often called Rusanov flux, this definition has different eigenvalues for each characteristic field. Thus, the cLLF flux is still close to the Roe flux with only slightly increased numerical dissipation. 


\subsubsection{Note on numerical dissipation}

The flux formulation given in Eq. 4 has two parts. The first term on the right-hand-side is the central flux term, and the second term is the dissipative flux term. Since $\mathbf{R}$ and $\mathbf{R}^{-1}$ are only forward and backward coordinate transformations, the dissipative flux merely depends on $\boldsymbol{\Lambda}$. Again, two different contributions to the dissipative flux can be distinguished. The advection dissipation is proportional to $|u|$, while the acoustic dissipation is proportional to $|u \pm c|$. This distinction might be helpful to understand the proposed cure of the shock instability.

\section{A modified flux formulation}

We relate the grid-aligned shock instability to another well-known difficulty of Godunov schemes in the low Mach number limit. The connection can be deduced from the observation that the instability only occurs when the shock front moves aligned with the computational grid. For Cartesian grids, this situation involves vanishing but non-zero velocity components in spatial directions other than the shock propagation direction for cells covering the shock front. Note that a perfect alignment leads to a purely one dimensional situation and no instability occurs. A small deflection is always required to trigger the instability.

The asymptotic analysis on the Roe flux and general Godunov schemes by Guillard et al. [29, 30] revealed that the centered terms of the flux formulations are of order $\mathcal{O}\left(1 / M^{2}\right)$, while the dissipative terms are of order $\mathcal{O}(1 / M)$ for low Mach numbers. Thus, even if the initial pressure contains

fluctuations of order $\mathcal{O}\left(M^{2}\right)$, the dissipative flux term will lead to pressure 
fluctuations of order $\mathcal{O}(M)$ when $M \rightarrow 0$. These pressure fluctuations are mostly of acoustic origin due to the fact that, for vanishing Mach numbers, the acoustic contribution to the numerical dissipation is much larger than that of the advection.

The main goal of the proposed modifications therefore is to balance the advective and acoustic dissipation in the low Mach number limit.

\subsection{A modified Roe-M flux}

A straightforward way to decrease the imbalance of advective and acoustic dissipation and consequently to avoid the amplification of present pressure disturbances is to increase the Mach number value $M$ in $\boldsymbol{\Lambda}$.

In case of the Roe flux, this can either be done by decreasing the acoustic dissipation

$$
\lambda_{1,5}^{\prime}=\hat{u} \pm \min (\phi|\hat{u}|, \hat{c}), \quad \lambda_{2,3,4}^{\prime}=\hat{u}
$$

or by increasing the advection dissipation

$$
\lambda_{1,5}^{\prime \prime}=u^{\prime \prime} \pm \hat{c}, \quad \lambda_{2,3,4}^{\prime \prime}=u^{\prime \prime}, \quad u^{\prime \prime}=\operatorname{sgn}(\hat{u}) \cdot \max \left(\frac{\hat{c}}{\phi},|\hat{u}|\right)
$$

where $\phi$ is a positive number of order $\mathcal{O}(1)$. Thus, the Mach number is limited to a value of $1 / \phi$. It is important to notice that both formulations do not change the eigenvector matrix nor the central flux term, although both modifications lead to comparable acoustic and advection contributions to the numerical dissipation in the low Mach number direction of the flow. While the Roe flux with Eq. (12) increases the total local numerical dissipation, the Roe flux with Eq. (11) decreases it. In the context of globally low Mach 
number flows, $\mathrm{Li}$ and $\mathrm{Gu}$ [32] developed a similar procedure. They proposed an All-Speed-Roe scheme that involves a momentum interpolation in the central flux term and a more complex adjustment of the sound speed in the low Mach number limit with similar effect as Eq. (11). Here, a stabilizing momentum interpolation is not required since the purpose of our method is to cure grid-aligned shock instabilities which never occur in flows with globally low Mach number.

There are many different methods in literature that cure the shock instability by increasing the numerical dissipation. To the knowledge of the authors, however, there is no approach that achieves this goal by further reducing the dissipation of the already low-dissipation Roe flux. Thus, we will focus on the modification with eigenvalues determined by Eq. (11) throughout the paper, even though both formulations by Eq. (11) or Eq. (12) are effective to prevent the grid-aligned shock instability. The Roe flux with low Mach number treatment will be denoted Roe-M in the following.

\subsection{A modified componentwise LLF-M flux}

The same procedure of decreasing the acoustic dissipation applied to the componentwise local Lax-Friedrichs flux leads to eigenvalues

$$
\begin{aligned}
& \left|\lambda_{1}^{\prime}\right|=\max \left(\left|u_{i}-c_{i}^{\prime}\right|,\left|u_{i+1}-c_{i+1}^{\prime}\right|\right), \\
& \left|\lambda_{2,3,4}^{\prime}\right|=\max \left(\left|u_{i}\right|,\left|u_{i+1}\right|\right), \\
& \left|\lambda_{5}^{\prime}\right|=\max \left(\left|u_{i}+c_{i}^{\prime}\right|,\left|u_{i+1}+c_{i+1}^{\prime}\right|\right),
\end{aligned}
$$

with

$$
c_{i, i+1}^{\prime}=\min \left(\phi\left|u_{i, i+1}\right|, c_{i, i+1}\right) .
$$


The componentwise local Lax-Friedrichs flux with low Mach number treatment will be denoted cLLF-M in the following.

\section{Properties of the modified flux}

In the following, a plane shock propagation along a rectangular duct with a defined disturbance level parallel to the shock propagation is considered to demonstrate the capabilities of the modified Roe flux, and to show the impact of the parameter $\phi$ on the shock stability property.

This test case was introduced by Quirk [12] as a minimal working example for the odd-even decoupling phenomenon. Despite its simplicity it is an efficient and reliable way to trigger the instability in combination with different numerical flux formulations. The rise of the instability can easily be investigated both qualitatively and quantitatively. The shock propagation is simulated on a domain of $[0,2400] \times[0,20]$ discretized with $2400 \times 20$ cells. Pre-shock density and pressure are set to unity, and all velocity components are set to zero. Originally, the disturbance was triggered by an odd-even offset of the center-line grid position, whereas we follow the approach of Kemm [33] and introduce artificial numerical noise to the primitive variables in the initial state since the computations are done with a Cartesian-coordinate formulation. Inflow and outflow conditions are applied at the left and at the right boundary, respectively. Symmetry boundary conditions, which are equivalent to reflecting wall conditions, are enforced both at the top and at the bottom boundary of the domain. We have performed simulations with the original $M a=6$ setup and with a more challenging $M a=20$ setup with initial conditions given by 


$$
(\rho, u, v, p)= \begin{cases}(1,0,0,1) & \text { if } x>5.0 \\ \left(\frac{216}{41}, 35 \frac{\sqrt{35}}{36}, 0, \frac{251}{6}\right) & \text { else (for } M a=6 \text { case), } \\ \left(\frac{160}{27}, \frac{133}{8} \sqrt{1.4}, 0,466.5\right) & \text { else (for } M a=20 \text { case), }\end{cases}
$$

where the shock front is placed initially at $x=5$. In order to monitor the growth rate of the disturbance quantitatively over time the maximum magnitude of the y-velocity component is evaluated in the whole domain as a measure of the deviation from the one-dimensional solution.

Fig. 1 shows the evolution of the velocity deviation for the $M a=6$ case when all initial primitive variables are superposed by uniform random perturbations ranging from $-0.5 \cdot 10^{-3}$ to $0.5 \cdot 10^{-3}$.

Color maps of density and velocity for different flux formulations are presented in Fig. 2. Note that minimum and maximum color values are chosen with respect to extreme values within the carbuncle.

As expected, the classical Roe flux shows an exponential instability that results in a carbuncle-like distortion of the shock front, see Fig. 2 left. The instability manifests itself most prominently by a significant rise of the velocity magnitude in the carbuncle region. The componentwise local Lax-Friedrichs flux behaves similarly as the Roe flux, with the difference that the simulation does not break due to a floating point exception. Instead the disturbance level remains bounded after amplification by three orders of magnitude until the end of the simulation, see Fig. 2 center. All Roe-type schemes with low Mach number treatment behave stable. Values of $\phi$ within a reasonable range between 1 and 10 show comparable results. Note that the simulation is performed up to a very late point in time, where the shock front, given in 


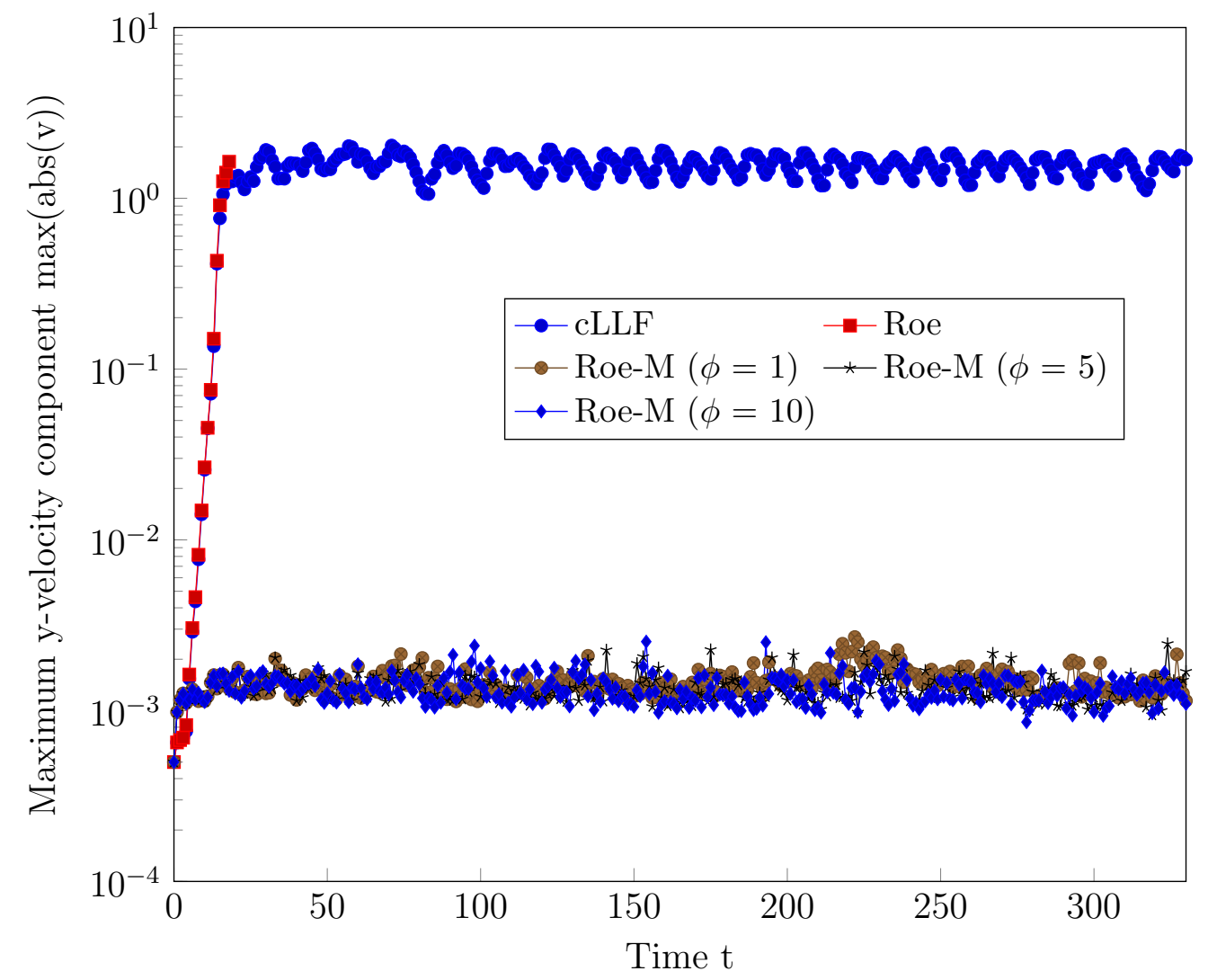

Figure 1: Instability progress in Quirk's test case with $M a=6$.
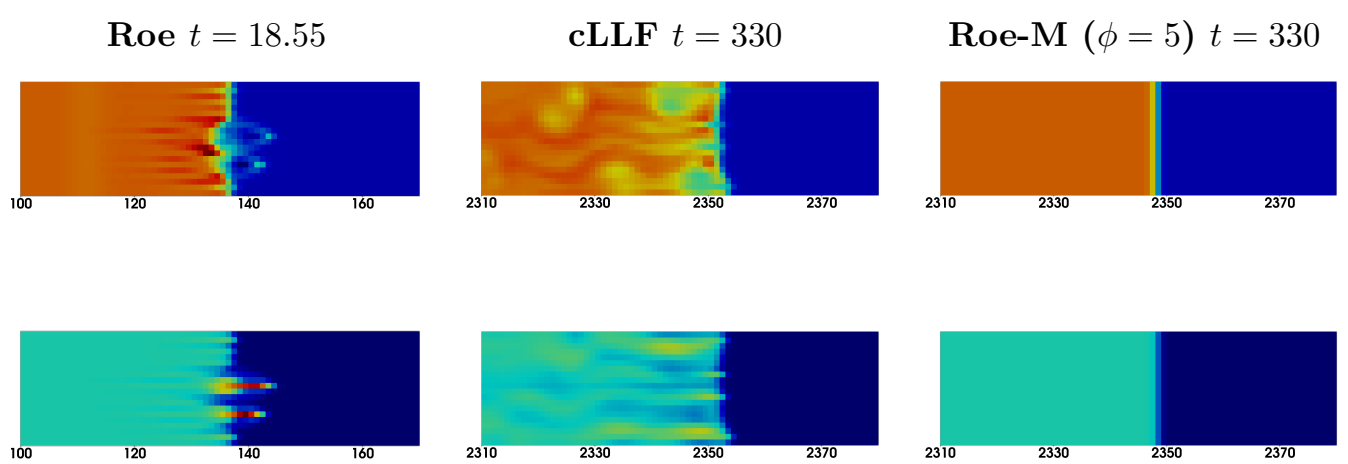

Figure 2: Quirk's test case with $M a=6$, (top) color map of density from dark blue $=0.5$ to red $=6.8$, (bottom) color map of velocity magnitude from dark blue $=0$ to red $=14.5$. 


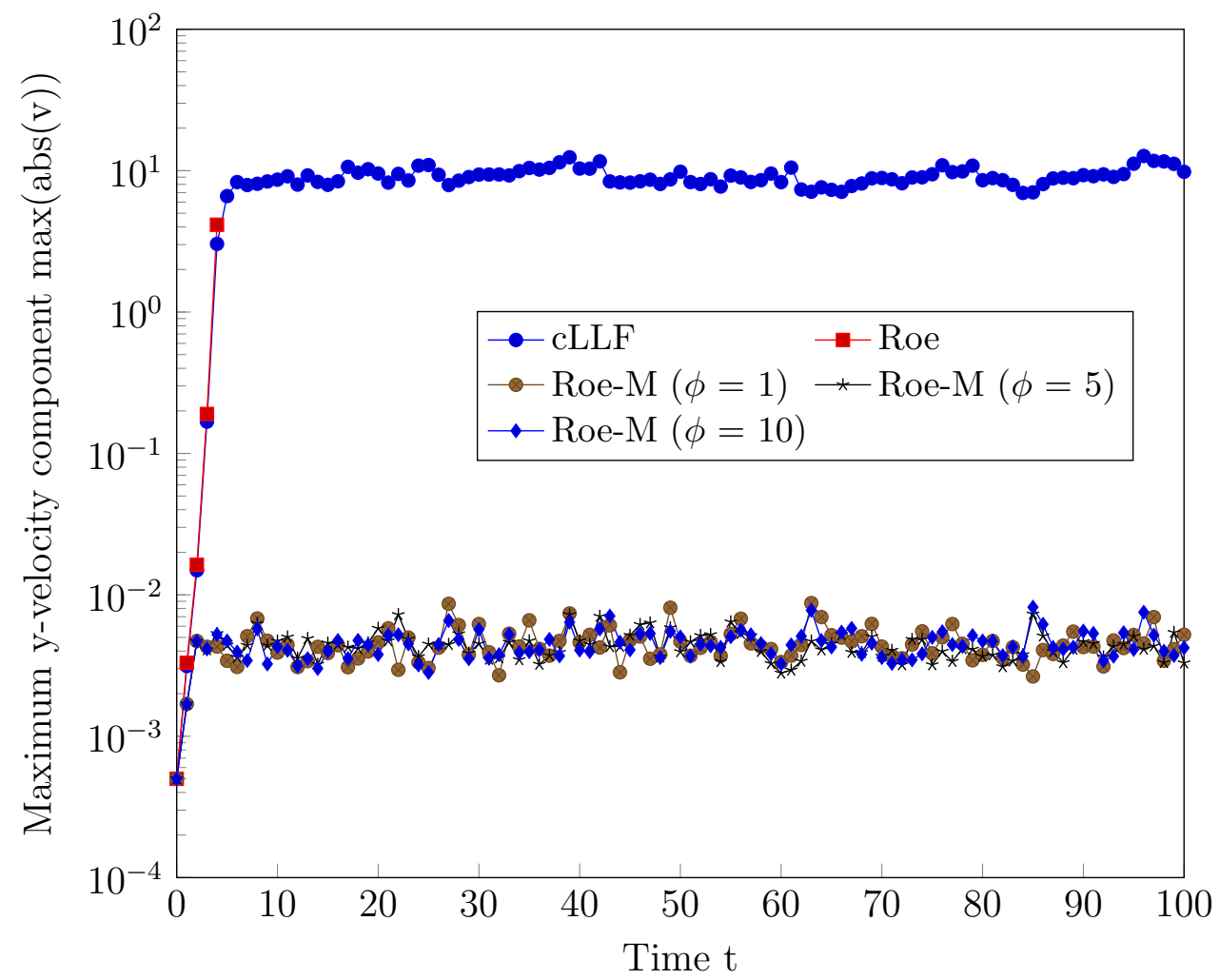

Figure 3: Instability progress in Quirk's test case with $M a=20$.

the right frame of Fig. 2, has already passed $x=2300$ without showing any non-physical behavior.

The same procedure has also been applied for a stronger shock with $M=$ 20. Figure 3 shows the resulting velocity deviations. The error growth rate for the classical Roe and componentwise local Lax-Friedrichs scheme increases noticeably, whereas deviations for the modified Roe fluxes remain bounded. The classical Roe scheme breaks after very few time steps for the increased Mach number. The final density distribution of the componentwise local LaxFriedrichs flux as shown in Fig. 4 reveals that the shock wave is not only 
$\mathbf{c L L F} t=100$

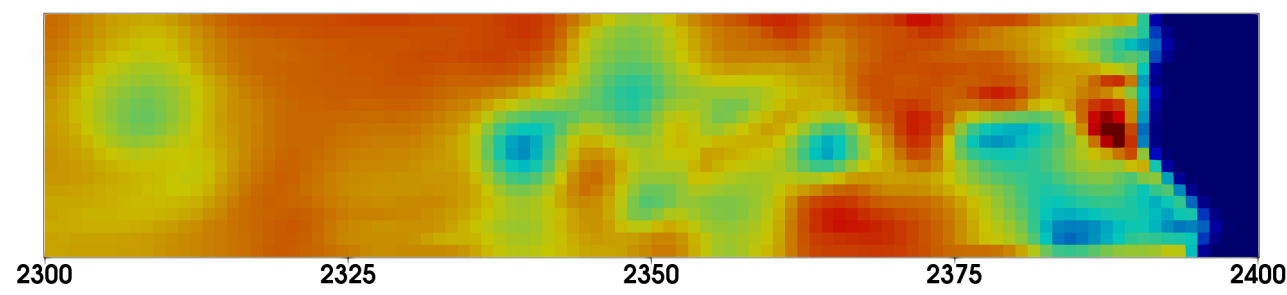

$\operatorname{Roe}-\mathbf{M}(\phi=5) t=100$

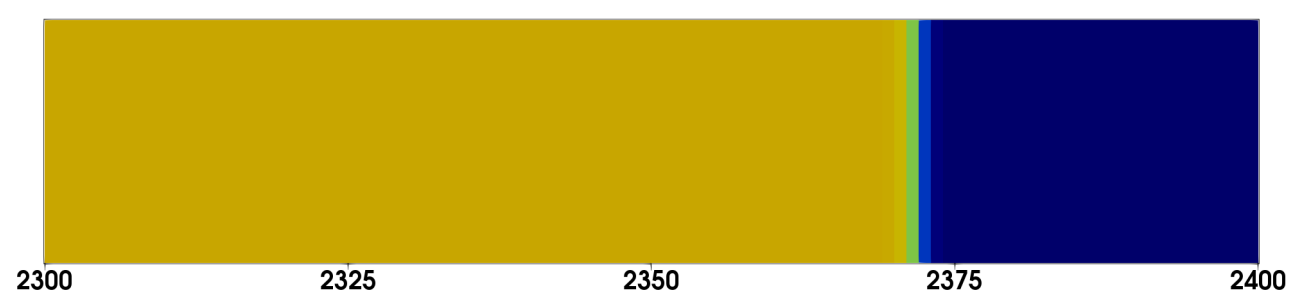

Figure 4: Quirk's test case with $M a=20$, color map of density from dark blue $=1.0$ to red $=8.0$.

severely disturbed, but also moves with an incorrect wave speed. Instead, the analytically expected position of the shock front is recovered by the modified Roe flux. Again, the result obtained by the modified scheme shows no sign of an instability.

\section{Note on the multidimensionality of the shock instability}

It is known that purely one-dimensional cases are not affected by the grid-aligned shock instability unless they are formulated in two dimensions. Following the argumentation of Sec. 3, this behavior can now be explained as we have identified the pressure fluctuation induced by the low Mach number of the transverse direction to be the driving force of the instability. The Roe approximation relies upon a finite-difference approach that consists of a 
dimension-by-dimension evaluation of the numerical flux contributions. This allows for a selective application of the low Mach number modification to fluxes in specific coordinate directions. Thus, it should be possible to cure the instability only along pre-selected coordinate directions. We expect the stability to be cured for shocks moving aligned to the grid in y-direction when the modified flux is applied in $\mathrm{x}$-direction and vice versa.

Due to its symmetry a Sedov blast wave problem is well-suited for demonstration. For this test case, a high pressure area with $P^{\text {inner }}=3.5 \cdot 10^{5}$ is initiated at the center of the domain that covers exactly one cell in each of the four quadrants. The rest of the domain is set to a near vacuum state with $P^{\text {outer }}=10^{-10}$. Density is set to unity and both velocity components are set to zero in the whole domain. Reflecting-wall conditions are applied at all boundaries. The domain is set to $[0,2.4] \times[0,2.4]$ with $480 \times 480$ cells.

Fig. 5 shows the pressure distribution at $t=0.1$ for both the original and the modified Roe scheme. While for the original Roe flux four distinct carbuncles have established at each location where the shock front moves aligned to the grid, the Roe-M flux is able to prevent the occurrence of the carbuncles effectively and maintains a sharp shock front everywhere. Now, the low Mach number modification is only applied for fluxes in x-direction, respectively in y-direction. The results given in Fig. 6 confirm exactly our expectations.

The cure of the flux in one direction affects the stability of shocks moving in the other direction. Actually, the application of the modification in $y$ direction is sufficient to stabilize the odd-even decoupling case presented in Sec. 4 where the shock front moves in x-direction. Three-dimensional 
Roe

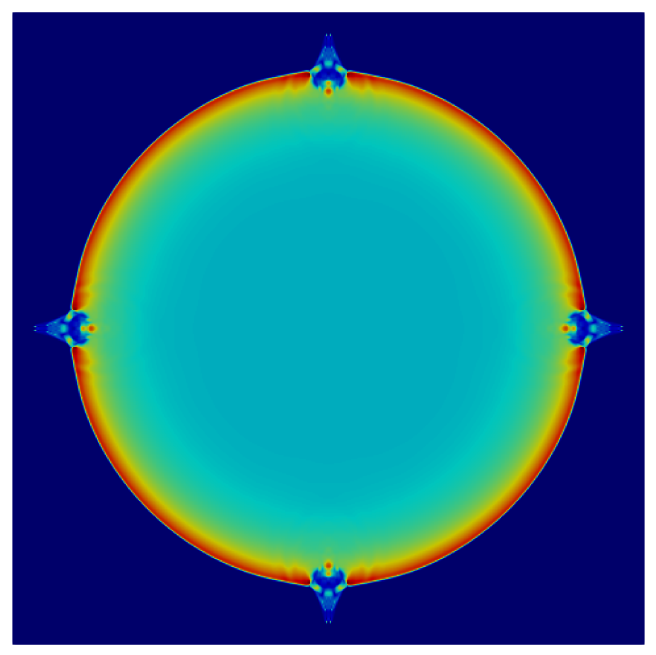

Roe-M

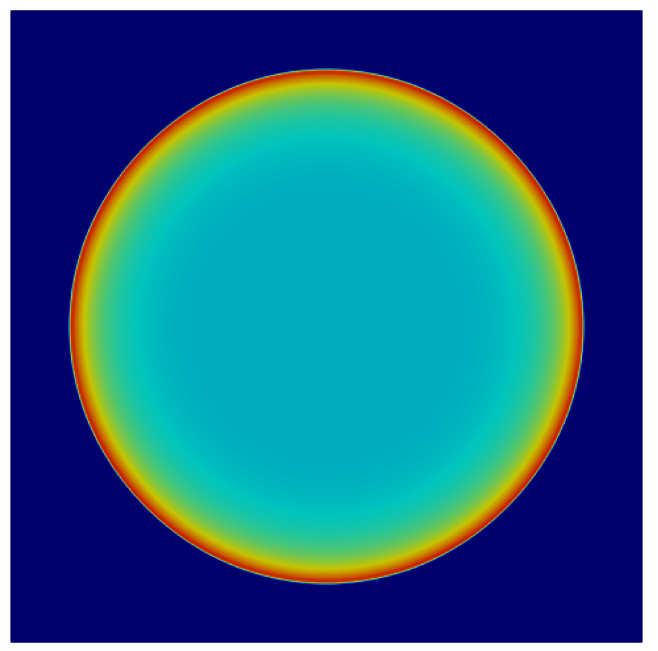

Figure 5: Two dimensional Sedov blast wave at $t=0.1$ : color map of pressure from blue $=0$ to red $=22.0$.

Roe-M (x-only)

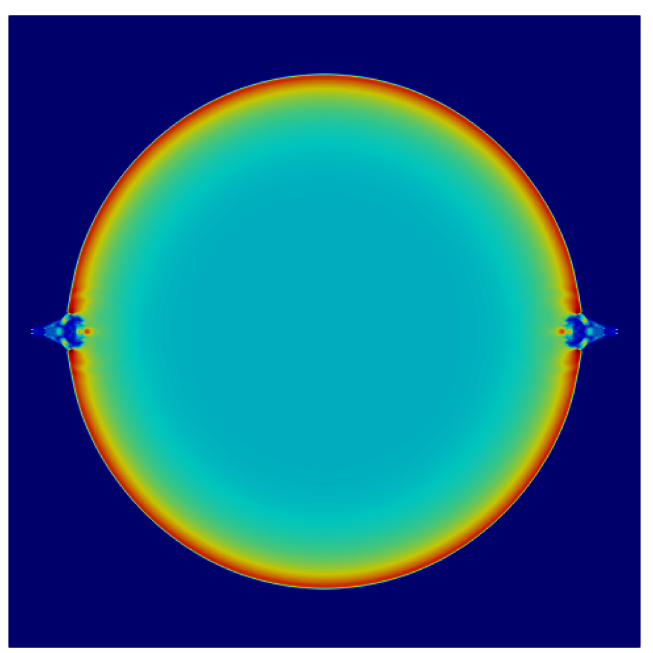

Roe-M (y-only)

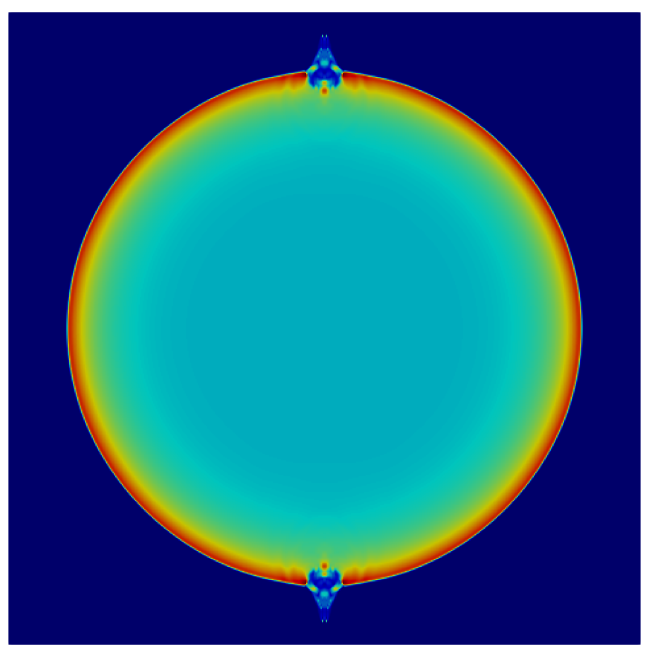

Figure 6: Two dimensional Sedov blast wave at $t=0.1$ : color map of pressure from blue $=0$ to red $=22.0$. 
calculations of the the Sedov blast wave show stable results only when the flux modification is applied at least to both other directions. Thus, the gridaligned shock instability is a true multidimensional phenomenon.

\section{Numerical results}

In this section, we investigate a wide range of test problems that are known to suffer from the shock instability. Together with Quirk's odd-even decoupling and the Sedov blast wave, which were presented in the previous sections, the classical set of test cases consists of the double Mach reflection problem [34], the 90 degree corner flow problem [12], the flow around a blunt body [13] and the "physical" carbuncle set-up of Elling [35]. Additionally, we will consider two multi-phase cases, where we encountered carbuncle-like instabilities during a shock-interface interaction both with air/helium and water/air.

If not mentioned otherwise, calculations were performed using the classi-

cal fifth-order WENO scheme [6] combined with a third-order strong-stabilitypreserving Runge-Kutta time integration [31]. Whenever the problem does not rely on positivity preservation, we apply a Roe flux [11] and its low Mach number modification, otherwise the componentwise local Lax-Friedrichs [6] and the corresponding low Mach number modification are employed. The $\phi$ value is always set to 5 . A level-set approach [36] is applied for multi-phase cases. The CFL number is set 0.6 for single-phase cases and 0.4 for cases that involve the level-set approach. The material is modelled as ideal gas with $\gamma=1.4$. 
cLLF

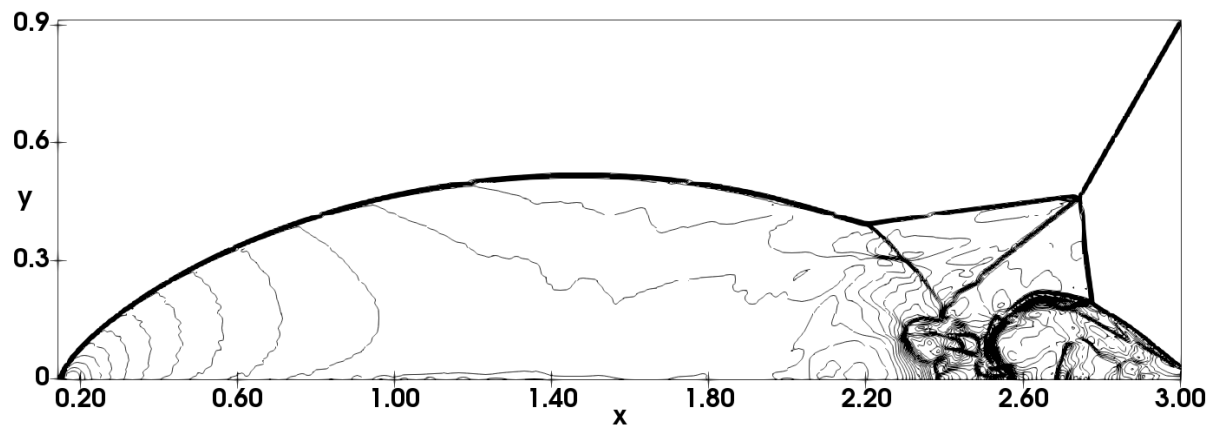

Roe-M

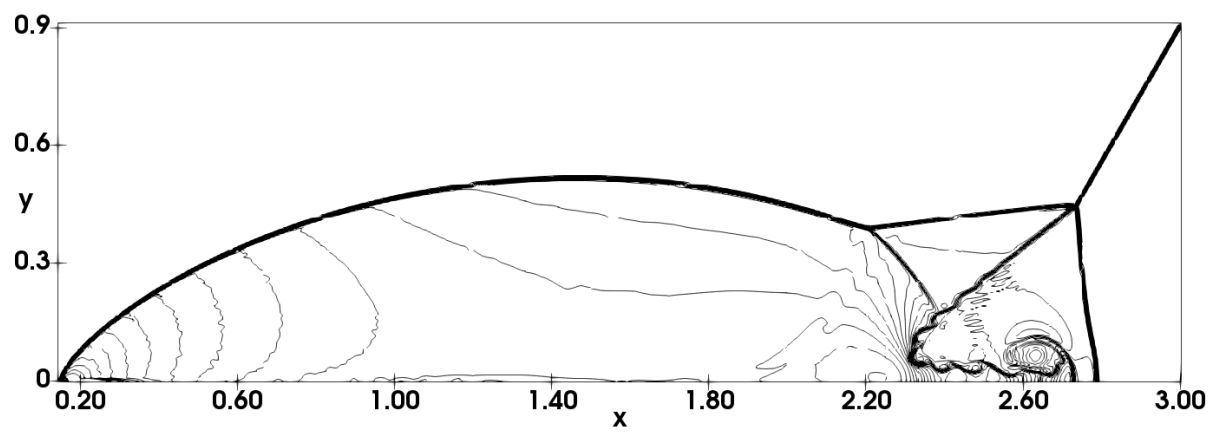

Figure 7: Double Mach reflection of a Mach 10 shock wave: 40 density contours from 1.88783 to 20.9144 at $t=0.2$

\subsection{Double Mach reflection problem}

The problem of Woodward and Colella [34] on the double Mach reflection is a popular benchmark test for the quality of a Riemann solver. It consists of a shock front that hits a ramp that is inclined by 30 degrees. Shock unstable schemes such as e.g. the Roe approximation may produce an artificially kinked Mach stem as described by various authors [12, 28, 33].

The initial conditions for a Mach 10 shock wave are 


$$
(\rho, u, v, p)= \begin{cases}(1.4,0,0,1) & \text { if } y<\sqrt{3}(x-1 / 6) \\ \left(8,33 \frac{\sqrt{3}}{8},-4.125,116.5\right) & \text { else, }\end{cases}
$$

and the final time is set to $t=0.2$. While the computational domain typically is chosen as $[0,4] \times[0,1]$, here, we use an even larger domain of $[0,4] \times[0,7]$ to avoid any boundary effects of the shock wave moving along the upper boundary. The domain is discretized with $960 \times 1680$ cells. A Neumann boundary condition with zero gradients for all variables is applied at the left, right and upper boundary. Along the bottom boundary, at $y=0$, the region from $x=0$ to $x=1 / 6$ is always assigned post-shock conditions, whereas reflecting-wall conditions are imposed from $x=1 / 6$ to $x=4$.

The problem of the kinked Mach stem is usually presented for low-order schemes. However, high-order schemes are likewise affected if the resolution is increased. The calculations are carried out with a fifth-order WENO-Z [37] scheme. Note, that the classical Roe flux is not able to deliver numerically stable results due to the instability at the leading Mach stem. Thus, the reference result is obtained using the componentwise local Lax-Friedrichs scheme. As shown in the top frame of Fig. 7, the componentwise local LaxFriedrichs flux suffers from a kinked Mach stem, a typical configuration of the carbuncle phenomenon. The modified Roe flux with even less numerical dissipation than the original Roe flux is able to produce numerically stable and correct results, see bottom of Fig. 7 . 

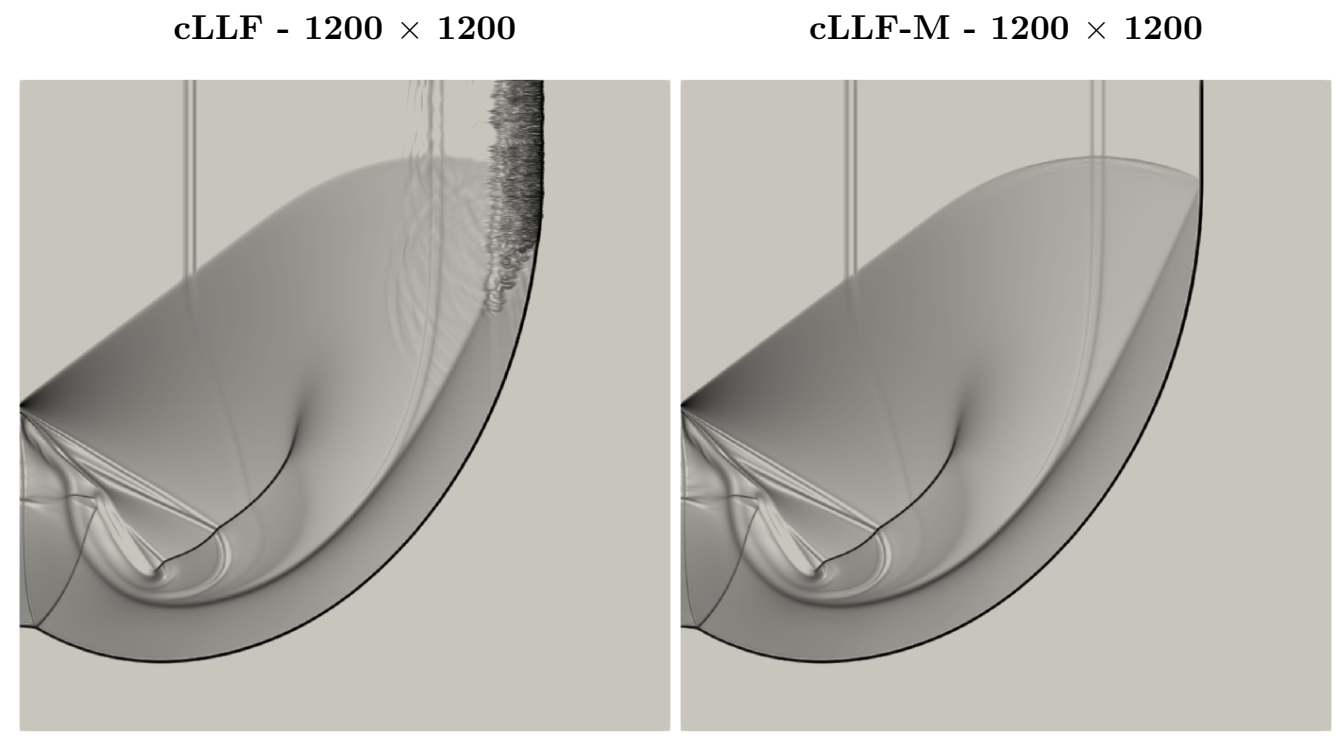

Figure 8: Corner diffraction of a Mach 5.09 shock wave: logarithmic gradients of density from 2 to 1,000 at $t=0.15717$

\subsection{Supersonic corner flow}

Another well-known test case, which was already described by Quirk [12] in the context of shock instability, is the diffraction of a shock wave around a sharp corner. This problem yields complicated flow patterns and many schemes encounter the situation of odd-even decoupling in parts of the flow where the shock wave is aligned to the grid. The instability occurs in particular when the mesh is highly refined.

We use a domain of size $[0,1] \times[0,1]$, that is uniformly initialized with $(\rho, u, v, p)=(1,0,0,1 / 1.4)$. Reflecting-wall boundary conditions are set everywhere, except of the upper left boundary at $x=0$ from $y=0.5$ to $y=1$. Here, the post-shock condition of a Mach 5.09 shock wave is prescribed. The final time is set to $0.8 / \mathrm{Ma}$. The original Roe flux is known to produce both 


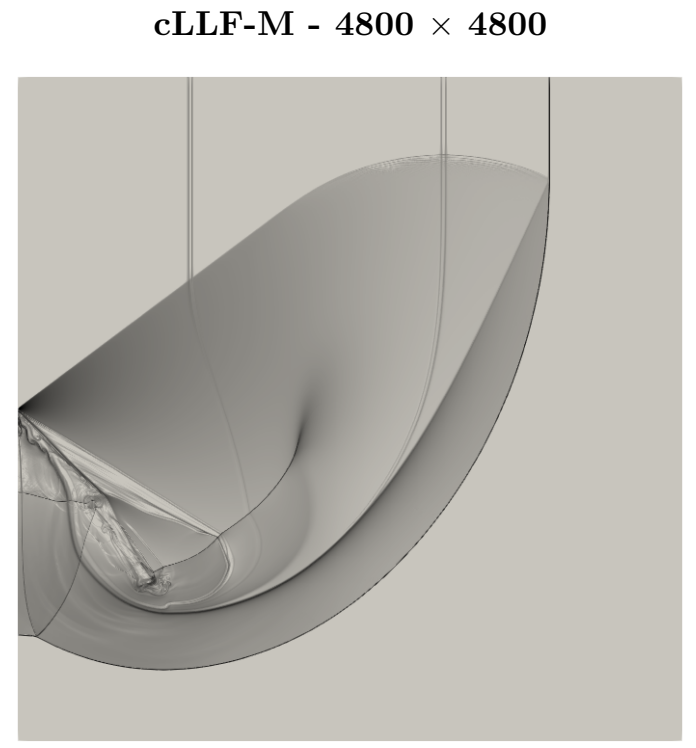

Figure 9: Corner diffraction of a Mach 5.09 shock wave (high resolution): logarithmic gradients of density from 2 to 1,000 at $t=0.15717$

numerically unstable results due to the positivity violation at the cells near the corner and non-physical results due to a rarefaction shock wave that establishes in the flow behind the corner [12]. Thus, the reference solution is again obtained using the componentwise local Lax-Friedrichs flux combined with a third-order WENO scheme [6].

The left frame of Fig. 8 shows a schlieren image of density gradients at the end of the simulation. The non-physical odd-even decoupling along the upper part of the shock front is clearly visible. Since the modified Roe flux only affects the shock instability and does not improve the positivity of the scheme, numerically stable results cannot be expected. However, correct physical results are obtained for the shock front when the modified componentwise local Lax-Friedrichs flux is applied, see Fig. 8 right. Note that 
the flow structure in the rest of the domain is not affected. The stability of the modified componentwise local Lax-Friedrichs flux is demonstrated for a highly resolved grid with $4800 \times 4800$ cells. The flow field still does not encounter any non-physical behavior as shown in Fig 9 .

\subsection{Supersonic flow around cylinder}

The most widespread manifestation of the grid-aligned shock instability is the so-called carbuncle phenomenon. It was first described in [13] for a supersonic flow around a cylinder. This case is challenging for shock-capturing schemes not only due to the grid-alignment but also also due to the steadiness of the shock. Godunov-type schemes are known to encounter difficulties for slowly moving and steady shocks $[12,38]$. The supersonic flow around a cylinder is often simulated using polar coordinates and implicit time integration up to a steady state $[21,28]$. However, in the context of this paper, we are mainly interested in the grid aligned shock instability, i.e. the carbuncle phenomenon, for Cartesian grids. Therefore, we apply the same Cartesian framework with explicit time integration as used for the other cases being aware of that the dissipation introduced by the time integration might not be enough to reach a fully converged steady state and a small resolved level of fluctuations around the steady shock may remain. The circular reflectingwall condition representing the cylinder is approximated using a level-set approach [36]. The left and the remaining right boundary are set to inflow and outflow conditions, respectively. Top and bottom boundary conditions are set to Neumann boundary conditions with zero gradient for all variables. We investigated both a Mach 3 and a Mach 20 flow around the cylinder. The whole domain is initially set to $(\rho, u, v, p)=(1, \sqrt{1.4} \cdot M a, 0,1)$. The domain 
cLLF

80 Cells/D

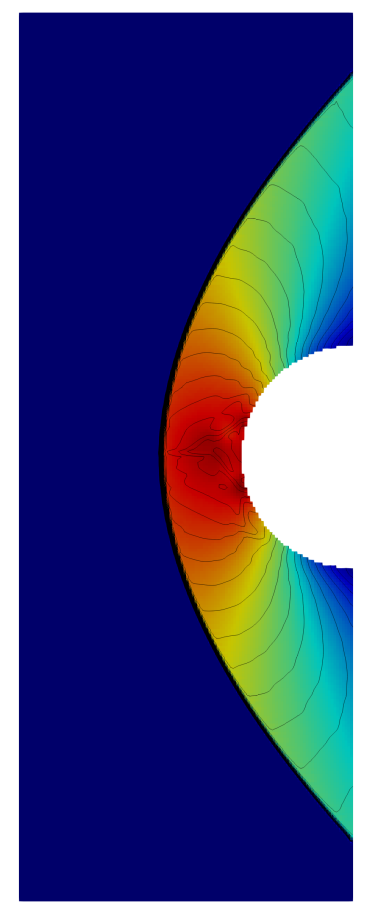

cLLF-M

80 Cells/D

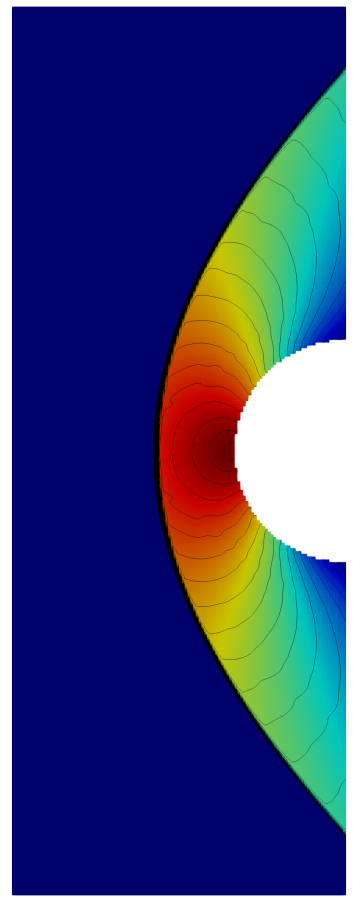

Figure 10: Supersonic flow around cylinder $M a=3$ at $t=1.5$ : color pressure map (blue $=1.0$ to red $=12.1$ ) is overlaid by 25 Mach contours (0.1 to 2.5 ).

size is chosen to $[0,0.3] \times[0,0.8]$ for the Mach 3 case and $[0,0.3] \times[0,0.6]$ for the Mach 20 case, where the center of the cylinder $(D=0.2)$ is placed in the center of the right boundary. Simulations have been performed using a resolution of 80 cells per diameter, which is comparable to what is used in literature [28].

The resulting pressure distributions for the low Mach number case are shown in Fig. 10 together with Mach contour lines that are chosen identical to [28]. The final time $t=1.5$ is large enough to ensure a fully developed bow 
cLLF

80 Cells/D

$t=0.06$

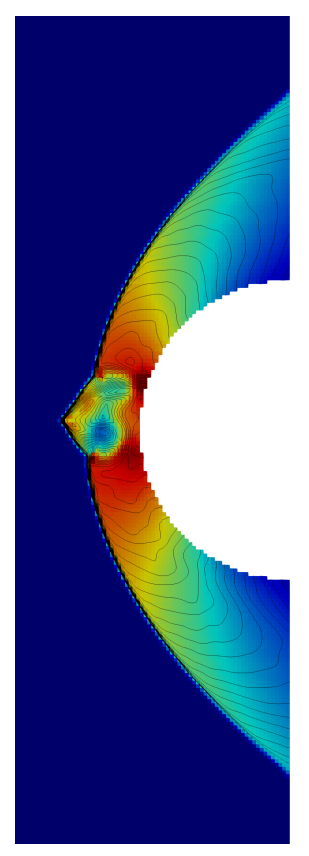

cLLF-M

80 Cells $/ D$

$t=0.06$

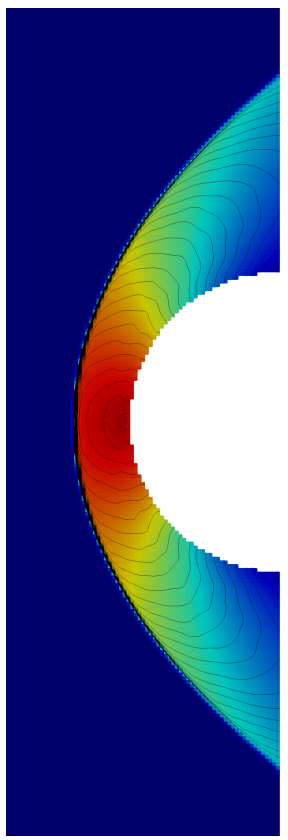

cLLF-M

80 Cells/D

$t=0.5$

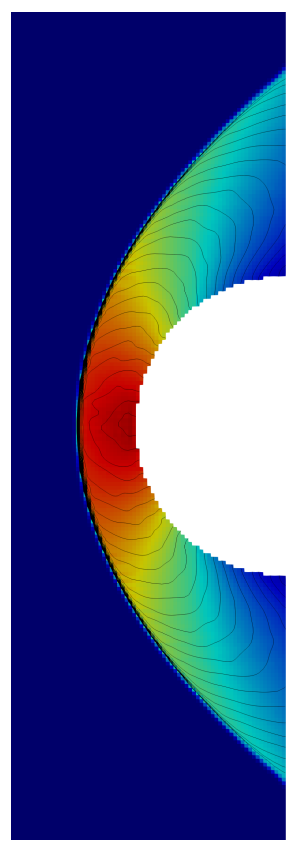

Figure 11: Supersonic flow around cylinder $M a=20$ : color pressure map (blue=1.0 to red $=550)$ is overlaid by 25 Mach contours (0.1 to 2.5$)$.

shock. The application of the original componentwise local Lax-Friedrichs flux results in a noticeably disturbed flow field in the region of the stagnation point. The modified componentwise local Lax-Friedrichs formulation damps the fluctuations considerably. Minor residual oscillations can be noticed in the pressure and velocity distribution.

To investigate the situation further, a more challenging Mach 20 flow is considered. The resulting pressure distributions are shown together with Mach contour lines in Fig. 11. Now the original componentwise local Lax- 
Friedrichs flux suffers clearly form the classical shock instability resulting in the carbuncle phenomenon. The instability already occurs while the bow shock establishes. For the same point in time the modified componentwise local Lax-Friedrichs flux provides stable results. The simulation remains stable until the final time of 0.5 is reached. The level of residual perturbations is slightly higher than in the low Mach number case.

\subsection{Elling test}

The numerical experiment described by Elling [35] provides a flow configuration where a carbuncle-like flow evolution can be physically justified and thus, it should be correctly recovered by the numerical flow solver. The test case consists of an interaction of a steady shock front with a vortex filament. For this specific condition, a carbuncle-like flow pattern has been observed in experiments [39]. Some of the shock stable schemes that suppress the instability by additional numerical dissipation, such as the HLLE solver, are not able to recover this physically valid carbuncle-like structure [33].

The problem is set up with a steady shock that is placed at $x=50$ in a domain of size $[0,100] \times[0,40]$, which is discretized by $1600 \times 640$ cells. The flow variables in the upstream region are set to $(\rho, u, v, p)=(1,1,0,5 / 63)$ and the downstream variables are set to $(\rho, u, v, p)=(27 / 7,7 / 27,0,155 / 189)$ corresponding to a steady Mach 3 shock. In the upstream flow the velocity is set to zero for $19.75<x<20.25$ imitating a vortex filament. Inflow and outflow conditions are applied at the left and right boundary, respectively. Reflecting-wall boundary conditions are set for the upper and lower boundary. The final time is set to 20 .

Note, that the filament in our case consists of eight cells compared to 
Roe $-1600 \times 640$

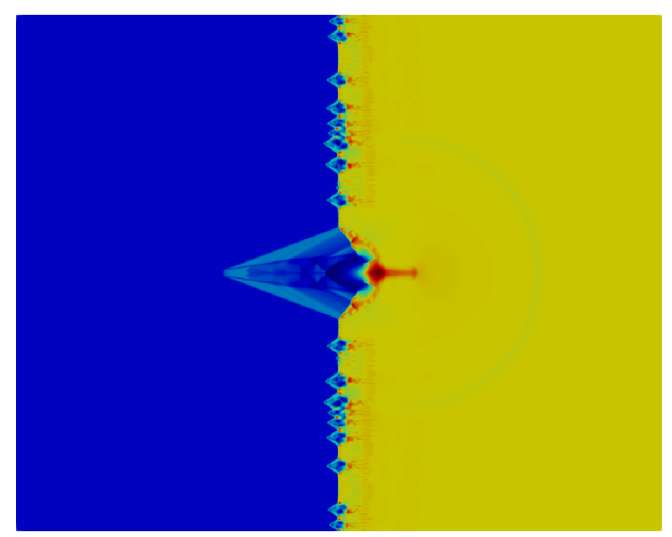

Roe-M - $1600 \times 640$

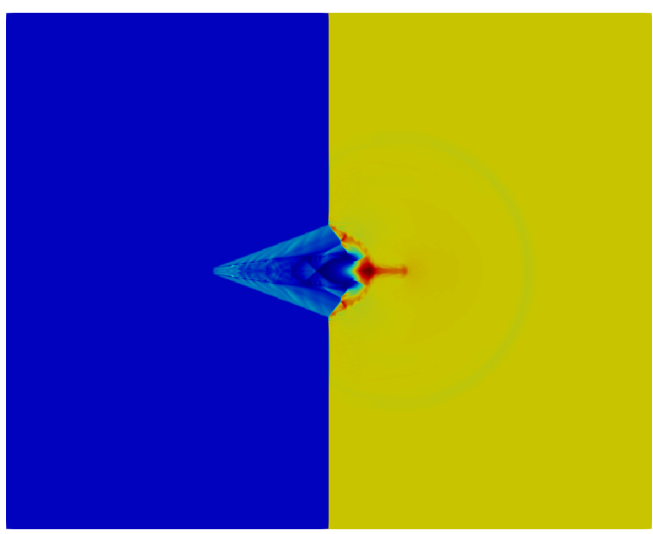

Figure 12: Elling test case with a vortex filament interaction with a steady Mach 3 shock: density contours from dark blue $=0.35$ to red $=6.0$ at $t=20$ for $x=[25,75]$

one cell in the original case. This change is necessary to stabilize the simulation when the Roe approximation is applied. The left frame of Fig. 12 shows the final result for the density profile when the original Roe flux is applied. Besides the expected carbuncle-like structure smaller carbuncles develop along the shock front. However, the modified Roe scheme is able to produce a disturbance-free shock front, while it does not suppress the "physical" carbuncle that is triggered by the vortex, see Fig. 12 right. The reduced numerical dissipation is clearly visible in the fine structure of the "physical" carbuncle.

\subsection{Shock interface interaction: helium bubble in air}

The grid-aligned shock instability does not only affect single-phase cases, but it may also limit the numerical investigation of shock-interface interaction problems. The following case investigates the interaction of a Mach 6 shock 


\section{Initial condition}

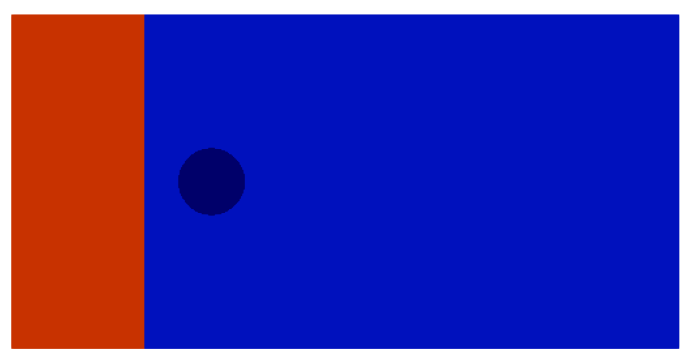

Figure 13: Initialization of shock interface interaction of a helium bubble in air: density contours from dark blue $=0.138$ to red $=6.5$

wave in air $(\gamma=1.4)$ with a helium bubble $(\gamma=1.66)$ similar to the setup in [1]. The helium bubble is placed at $x=0.15, y=0.125$ within in a domain of size $[0,0.5] \times[0,0.25]$. The initial diameter of the bubble is set to $D=0.05$. The shock front is initially placed at $x=0.1$. The resolution is set to $512 \times 256$. The pre-shock domain is at rest with $\rho^{A i r}=1, \rho^{H e}=0.138$ and $p^{\text {Air }}=p^{H e}=1$. The post-shock values of air are identical to the Mach 6 case given by Eq. (15). Inflow and outflow conditions are applied at the left and right boundary, respectively. Neumann boundaries with zero gradient for all quantities are set at the remaining boundaries. The initial setup is given in Fig. 13.

When the original Roe approximation is applied, three distinct carbuncles occur which deteriorate the flow field behind the shock wave massively as depicted in the top frame of Fig. 14. The modified Roe approximation is able to prevent the occurrence of the carbuncles effectively. The resulting flow evolution with stable shock front is shown in the bottom frame of Fig. 14. 


\section{Roe}

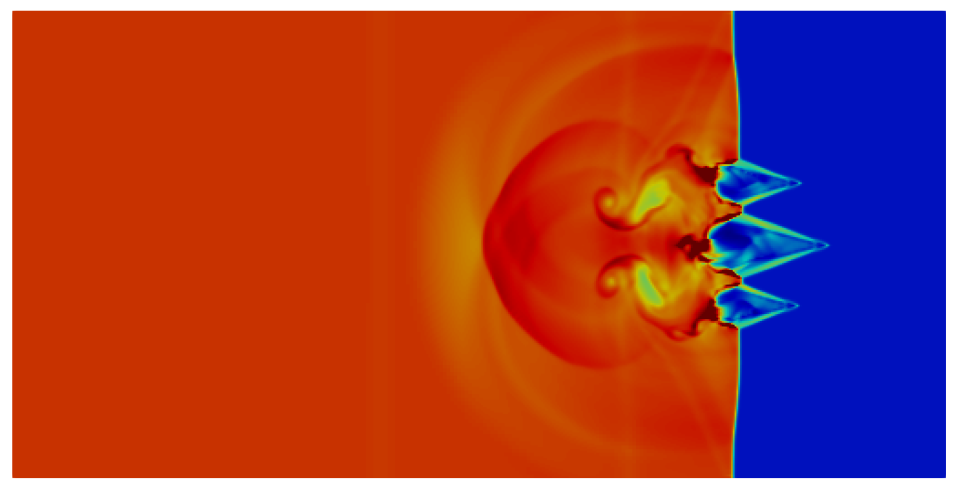

\section{Roe-M}

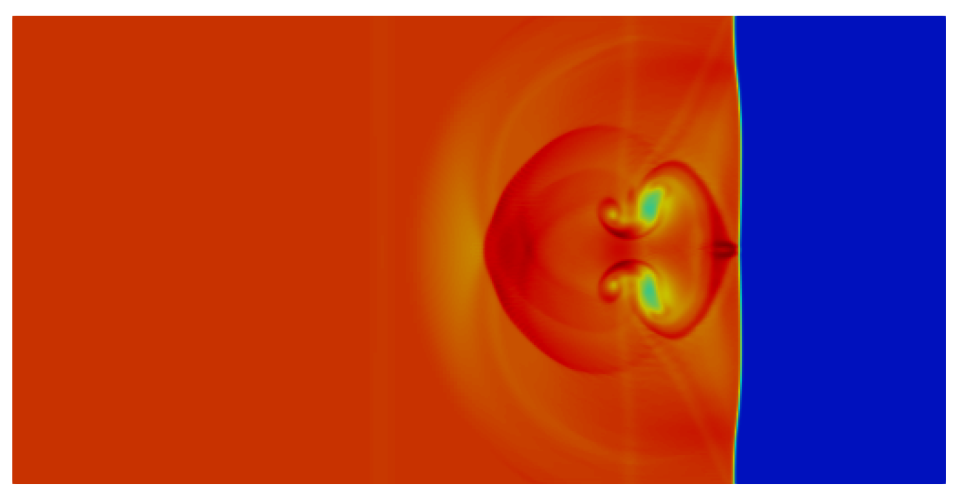

Figure 14: Shock interface interaction of a helium bubble in air at $t=0.04$ : density contours from dark blue $=0.138$ to red $=6.5$

\subsection{Shock interface interaction: air bubble in water}

Finally, another two-phase setup is investigated featuring a water-air setup with a large density ratio as described in [2]. As the shock hits the air bubble, a strong transmitted shock wave starts to travel within the air bubble. When low dissipation schemes such as the Roe approximation are applied, the shock front in the air bubble may suffer from the grid alignedshock instability. 
Roe

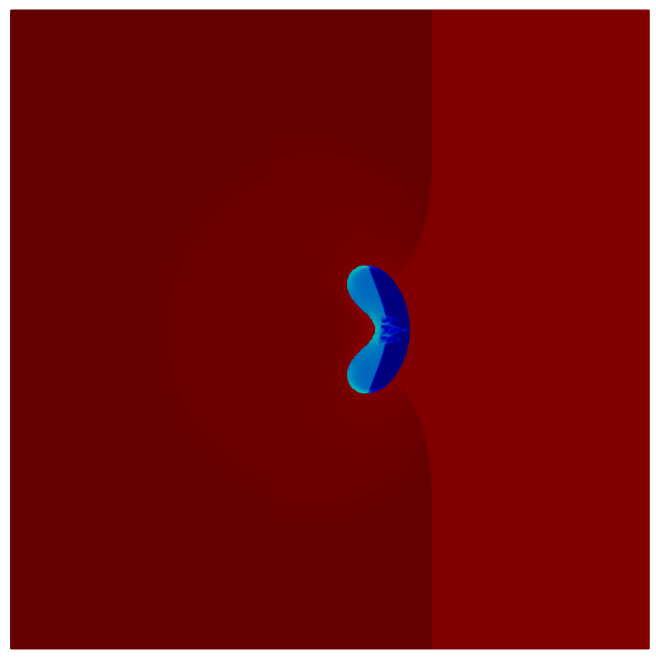

Roe-M

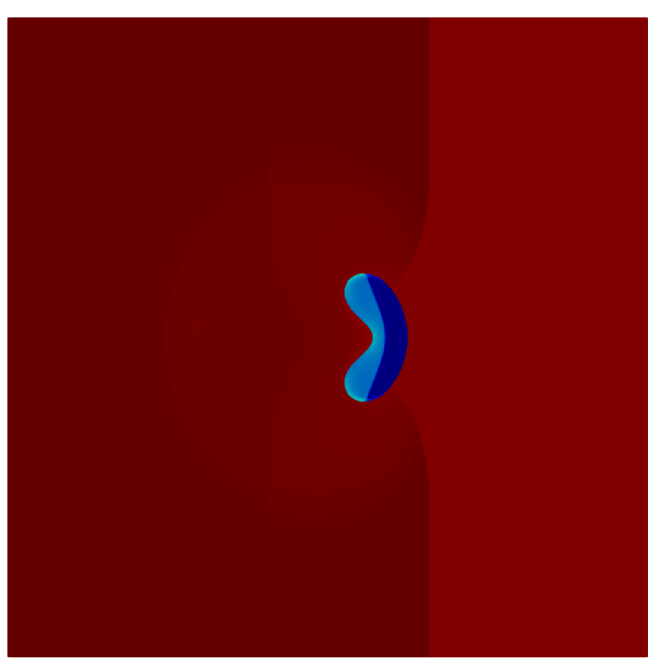

Figure 15: Shock interface interaction of a air bubble in water at $t=2.9 \cdot 10^{-6}$ : logarithmic color map of density from dark blue $=0.138$ to red $=6.5$.

We follow the setup of [2] with a domain size of $[0,0.024] \times[0,0.024]$, where the air bubble $(D=0.006)$ is placed in the middle of the domain.Inflow and outflow conditions are applied at the left and right boundary, respectively. Neumann boundaries with zero gradient for all quantities are set at the remaining boundaries. The shock front is initially placed at $x=0.008$. The initial condition are given by

$$
(\rho, u, v, p)= \begin{cases}\left(1323.65,661.81,0,1.6 \cdot 10^{9}\right) & \text { water post-shock } \\ \left(1000,0,0,10^{5}\right) & \text { water pre-shock } \\ \left(1,0,0,10^{5}\right) & \text { air, }\end{cases}
$$

where water is modelled with a stiffened equation of state $\left(\gamma=4.4, P_{\text {inf }}=\right.$ $\left.6 \cdot 10^{8}\right)$ and air as ideal gas $(\gamma=1.4)$. The resolution is set to $640 \times 640$. 

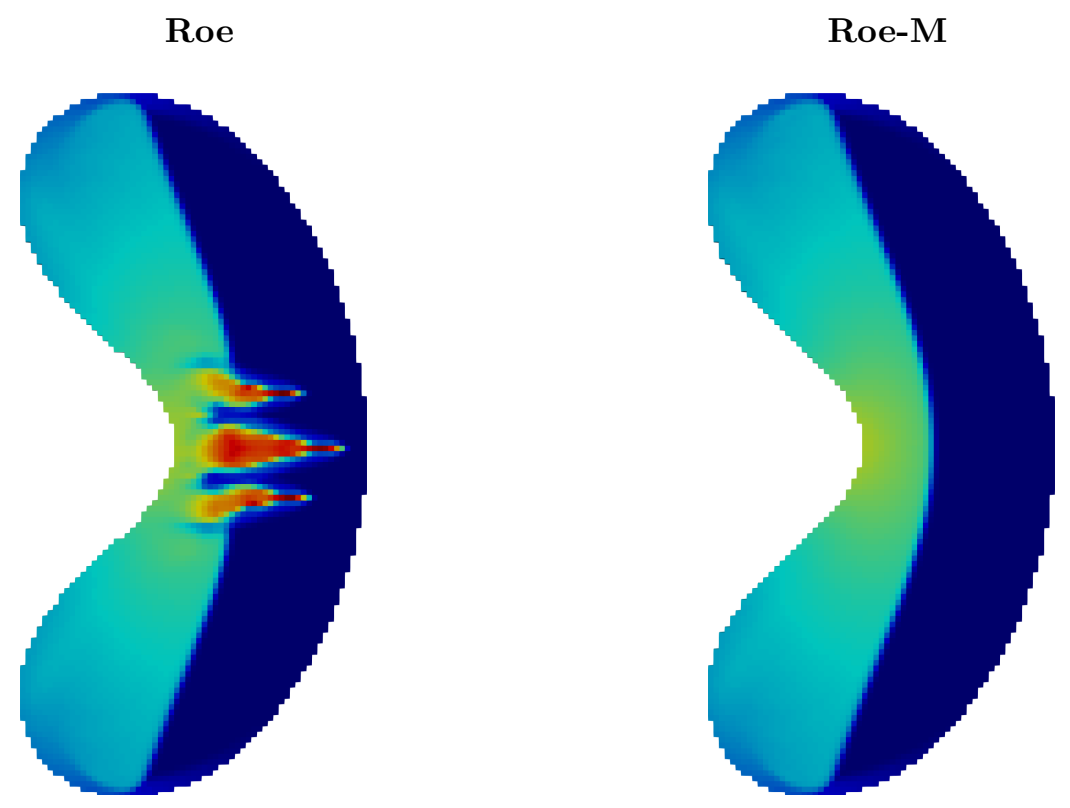

Figure 16: Shock interface interaction of a air bubble in water at $t=2.9 \cdot 10^{-6}$ : velocity magnitude within the air bubble from dark blue $=0$ to red $=4400$.

Fig. 15 shows the results for the density distribution for the whole domain, while Fig. 16 is focused on the velocity distribution in the air bubble at $t=2.9 \cdot 10^{-6}$. Again, the modified Roe flux is able to recover a sharp shock front without any instability effects.

\section{Conclusion}

In this paper, we have presented a procedure to obtain low-dissipation flux approximations that are stable against the grid-aligned shock instability and require only minor modifications to existing schemes. The procedure is motivated by connecting the grid-aligned shock instability to the well-known low Mach number effect of Godunov schemes. The typical setup for the instability consists of a shock front that propagates in one direction of the 
Cartesian grid, where the disturbances parallel to the front propagate in a low Mach number fashion. In this situation, the acoustic contribution to dissipation is dominant and leads to an amplification of pressure disturbances that causes the instability. The proposed procedure avoids the amplification and therefore cures the instability by limiting the ratio of the advection and the acoustic contribution to the dissipation term. Two possible implementations of the procedure were described in detail. Increasing the advection contribution to the dissipation term results in an increased overall dissipation, while reducing the acoustic dissipation leads to an decreased overall dissipation. A modified Roe approximation (Roe-M) and a modified componentwise local Lax-Friedrichs flux (cLLF-M) are given.

Results obtained with the low-Mach-number modified flux formulations for a comprehensive series of test cases confirm that the prime reason of the grid-aligned shock instability is not due to an insufficient amount of numerical dissipation, but due to the inadequate scaling of the dissipative flux contributions in the low Mach number limit. Moreover, we demonstrated that the shock instability can be cured by reducing the overall dissipation of the scheme. The preservation of high accuracy in all parts of the domain including the precise capturing of contact waves is therefore straightforward and does not require any further procedures. The Roe-M flux is stable against the grid-aligned shock instability, although it still exhibits other well-known deficiencies of the Roe flux such as lack of positivity and non-physical expansion shocks. The proposed method can be combined with entropy satisfying and positivity preserving techniques if required. The low Mach number treatment is most simple to apply to the Roe and componentwise local Lax- 
Friedrichs approximation, where only the calculation of the eigenvalues is affected. However, application to other flux formulations is also possible. Demonstrations of the Roe-M flux for two multi-phase applications including shock interactions with a helium bubble in air and an air bubble in water revealed excellent results and demonstrate that the proposed scheme is capable to simulate complex super- and hypersonic flow physics while maintaining high accuracy and robustness.

\section{Acknowledgements}

This project has received funding from the European Research Council (ERC) under the European Union's Horizon 2020 research and innovation programme (grant agreement No. 667483).

\section{References}

[1] A. Bagabir, D. Drikakis, Mach number effects on shock-bubble interaction, Shock Waves 11 (3) (2001) 209-218.

[2] O. Haimovich, S. H. Frankel, Numerical simulations of compressible multicomponent and multiphase flow using a high-order targeted ENO (TENO) finite-volume method, Computers \& Fluids 146 (2017) 105-116.

[3] E. F. Toro, Riemann solvers and numerical methods for fluid dynamics: a practical introduction, Springer Science \& Business Media, 2013.

[4] S. K. Godunov, A difference method for numerical calculation of discontinuous solutions of the equations of hydrodynamics, Matematicheskii Sbornik 89 (3) (1959) 271-306. 
[5] A. Harten, B. Engquist, S. Osher, S. R. Chakravarthy, Uniformly high order accurate essentially non-oscillatory schemes, III, Journal of Computational Physics 71 (2) (1987) 231-303.

[6] G.-S. Jiang, C.-W. Shu, Efficient implementation of weighted ENO schemes, Journal of Computational Physics 126 (1) (1996) 202-228.

[7] A. Harten, P. D. Lax, B. v. Leer, On upstream differencing and Godunov-type schemes for hyperbolic conservation laws, SIAM Review 25 (1) (1983) 35-61.

[8] B. Einfeldt, On Godunov-type methods for gas dynamics, SIAM Journal on Numerical Analysis 25 (2) (1988) 294-318.

[9] E. F. Toro, M. Spruce, W. Speares, Restoration of the contact surface in the HLL-Riemann solver, Shock Waves 4 (1) (1994) 25-34.

[10] S. Osher, F. Solomon, Upwind difference schemes for hyperbolic systems of conservation laws, Mathematics of Computation 38 (158) (1982) 339374.

[11] P. L. Roe, Approximate Riemann solvers, parameter vectors, and difference schemes, Journal of Computational Physics 43 (2) (1981) 357-372.

[12] J. J. Quirk, A contribution to the great Riemann solver debate, in: Upwind and High-Resolution Schemes, Springer, 1997, pp. 550-569.

[13] K. Peery, S. Imlay, Blunt-body flow simulations, in: 24th Joint Propulsion Conference, 1988, p. 2904. 
[14] R. Sanders, E. Morano, M.-C. Druguet, Multidimensional dissipation for upwind schemes: stability and applications to gas dynamics, Journal of Computational Physics 145 (2) (1998) 511-537.

[15] M. Pandolfi, D. D'Ambrosio, Numerical instabilities in upwind methods: analysis and cures for the carbuncle phenomenon, Journal of Computational Physics 166 (2) (2001) 271-301.

[16] J. Gressier, J.-M. Moschetta, Robustness versus accuracy in shock-wave computations, International Journal for Numerical Methods in Fluids 33 (3) (2000) 313-332.

[17] M.-S. Liou, Mass flux schemes and connection to shock instability, Journal of Computational Physics 160 (2) (2000) 623-648.

[18] M. Dumbser, J.-M. Moschetta, J. Gressier, A matrix stability analysis of the carbuncle phenomenon, Journal of Computational Physics 197 (2) (2004) 647-670.

[19] M.-S. Liou, A sequel to AUSM: AUSM+, Journal of Computational Physics 129 (2) (1996) 364-382.

[20] Y.-X. Ren, A robust shock-capturing scheme based on rotated Riemann solvers, Computers \& Fluids 32 (10) (2003) 1379-1403.

[21] S.-S. Kim, C. Kim, O.-H. Rho, S. K. Hong, Cures for the shock instability: Development of a shock-stable Roe scheme, Journal of Computational Physics 185 (2) (2003) 342-374. 
[22] S.-S. Chen, C. Yan, B.-X. Lin, Y.-S. Li, A New Robust Carbuncle-Free Roe Scheme for Strong Shock, Journal of Scientific Computing (2018) $1-28$.

[23] S. D. Kim, B. J. Lee, H. J. Lee, I.-S. Jeung, Robust HLLC Riemann solver with weighted average flux scheme for strong shock, Journal of Computational Physics 228 (20) (2009) 7634-7642.

[24] Z. Shen, W. Yan, G. Yuan, A robust HLLC-type Riemann solver for strong shock, Journal of Computational Physics 309 (2016) 185-206.

[25] S. Simon, J. Mandal, A simple cure for numerical shock instability in the HLLC Riemann solver, Journal of Computational Physics 378 (2019) 477-496.

[26] K. H. Kim, C. Kim, O.-H. Rho, Methods for the accurate computations of hypersonic flows: I. AUSMPW+ scheme, Journal of Computational Physics 174 (1) (2001) 38-80.

[27] K. Kitamura, E. Shima, Towards shock-stable and accurate hypersonic heating computations: A new pressure flux for AUSM-family schemes, Journal of Computational Physics 245 (2013) 62-83.

[28] A. V. Rodionov, Artificial viscosity in Godunov-type schemes to cure the carbuncle phenomenon, Journal of Computational Physics 345 (2017) 308-329.

[29] H. Guillard, C. Viozat, On the behaviour of upwind schemes in the low Mach number limit, Computers \& Fluids 28 (1) (1999) 63-86. 
[30] H. Guillard, A. Murrone, On the behavior of upwind schemes in the low Mach number limit: II. Godunov type schemes, Computers \& Fluids 33 (4) (2004) 655-675.

[31] S. Gottlieb, C.-W. Shu, E. Tadmor, Strong stability-preserving highorder time discretization methods, SIAM Review 43 (1) (2001) 89-112.

[32] X.-s. Li, C.-w. Gu, An all-speed Roe-type scheme and its asymptotic analysis of low Mach number behaviour, Journal of Computational Physics 227 (10) (2008) 5144-5159.

[33] F. Kemm, Heuristical and numerical considerations for the carbuncle phenomenon, Applied Mathematics and Computation 320 (2018) 596613.

[34] P. Woodward, P. Colella, The numerical simulation of two-dimensional fluid flow with strong shocks, Journal of Computational Physics 54 (1) (1984) 115-173.

[35] V. Elling, The carbuncle phenomenon is incurable, Acta Mathematica Scientia 29 (6) (2009) 1647-1656.

[36] X. Y. Hu, B. Khoo, N. A. Adams, F. Huang, A conservative interface method for compressible flows, Journal of Computational Physics 219 (2) (2006) 553-578.

[37] R. Borges, M. Carmona, B. Costa, W. S. Don, An improved weighted essentially non-oscillatory scheme for hyperbolic conservation laws, Journal of Computational Physics 227 (6) (2008) 3191-3211. 
[38] M. Bultelle, M. Grassin, D. Serre, Unstable Godunov discrete profiles for steady shock waves, SIAM Journal on Numerical Analysis 35 (6) (1998) 2272-2297.

[39] I. M. Kalkhoran, M. K. Smart, Aspects of shock wave-induced vortex breakdown, Progress in Aerospace Sciences 36 (1) (2000) 63-95. 